\title{
Treatment of displaced transverse fractures of the patella: modified tension band wiring technique with or without augmented circumferential cerclage wire fixation
}

Tien-Yu Yang ${ }^{1,2}$, Tsan-Wen Huang ${ }^{1,2}$, Po-Yao Chuang ${ }^{1,2}$ and Kuo-Chin Huang ${ }^{1,2,3^{*}}$ (i)

\begin{abstract}
Background: Displaced transverse fractures of the body of the patella are usually associated with disruption of extensor mechanism and should be fixed surgically. The most common method is a tension band wiring (TBW) technique. Some surgeons concurrently employ an augmented circumferential cerclage wiring (ACCW) technique to help fracture stabilization and aid in fracture healing; however, its role and effect on the treatment outcomes is unclear.

Methods: We performed a STROBE-compliant retrospective observational cohort study on all cases of acute closed patella fracture that were treated at our institution between 2006 and 2012. Of 185 episodes, 72 (38.9\%) were eligible for this study according to our inclusion/exclusion criteria. We classified these subjects with AO/OTA type 34-C1.1 or 34-C2 lesions into two groups for analyses: fractures treated with modified TBW and ACCW (group 1, $n=27$ ) and those treated with modified TBW alone (group 2, n=45). Plain radiographs were used to evaluate radiographic outcomes and the effect of potential risk factors on fixation failure was analyzed by subgroup comparisons.

Results: Our results revealed that there were no significant differences in the rates of fixation failure $(P=0.620)$, nonunion $(P=0.620)$, and revision surgery $(P=0.620)$ between the groups. Although not statistically significant, there was a trend towards a positive risk association between fixation failure and age distribution $>60$ years $(10.0 \% \mathrm{vs} .0 .0 \%$, $P=0.124 ; \mathrm{OR}=8.0, P=0.168)$ and $>70$ years $(9.4 \%$ vs. $2.5 \%, P=0.321 ; \mathrm{OR}=4.0, P=0.237)$ and the superficial level of the K-wires (12.0\% vs. 1.5\%, $P=0.117 ; \mathrm{OR}=6.3, P=0.121)$. Regarding those modified TBW patients concurrently treated with an ACCW, the potential risk association between fixation failure and the superficial level of the K-wire was prone to increase further ( $28.6 \%$ vs. $0.0 \%, P=0.060 ; \mathrm{OR}=18.6, P=0.071$ ).

Conclusions: Concurrent application of an ACCW might be needless and not efficacious to help fracture stabilization and healing in patients having been treated with modified TBW for displaced transverse fractures of the body of the patella. Adherence to correct surgical technique such as putting the K-wires at the proper level and securing control of the both ends of the K-wires may be more important and help in improving outcomes.
\end{abstract}

Keywords: Patella fracture, Tension band wiring, Circumferential cerclage wiring, Fixation failure, Nonunion

\footnotetext{
* Correspondence: kc2672@gmail.com

${ }^{1}$ Chang Gung University College of Medicine, Taoyuan, Taiwan

2Department of Orthopaedic Surgery, Chang Gung Memorial Hospital, Chiayi,

Taiwan

Full list of author information is available at the end of the article
}

(c) The Author(s). 2018 Open Access This article is distributed under the terms of the Creative Commons Attribution 4.0 International License (http://creativecommons.org/licenses/by/4.0/), which permits unrestricted use, distribution, and reproduction in any medium, provided you give appropriate credit to the original author(s) and the source, provide a link to the Creative Commons license, and indicate if changes were made. The Creative Commons Public Domain Dedication waiver (http://creativecommons.org/publicdomain/zero/1.0/) applies to the data made available in this article, unless otherwise stated. 


\section{Background}

Among all fracture types of the patella, transverse fracture of the body is the most common [1-4]. If displaced, it should be repaired surgically to restore the function of extensor mechanism and aid in fracture healing $[5,6]$. Tension band wiring (TBW) technique, one of the most commonly used surgical method in treating a tension fracture [7-10], has a sound biomechanical advantage such that it can convert a tensile force into a compressive force when the joint is brought through a range of motion [11-13]. With appropriate application of this technique to treat the displaced transverse fractures of the patella, the security of fixation would allow early joint mobilization and the prevention of knee stiffness $[14,15]$. The rate of fixation failure is not high and serious complications are uncommon [16]. Despite the potential occurrence of subjective complaints due to hardware irritation, TBW remains the gold standard for the treatment of displaced transverse fractures of the patella body $[2,3,7,8,14-17]$. At our institution, a modified TBW technique involving bending both ends of the K-wires is used regularly. Using this modified technique, $\mathrm{Wu}$ et al. had reported an excellent treatment result with smooth fracture union, good recovery of joint motion, and few complaints of hardware irritation (3\%) [15].

Even though the treatment results of TBW for displaced transverse patella fractures are promising, some surgeons recommend that the combination of augmented circumferential cerclage wiring (ACCW) and TBW would lead to a greater strength of fixation and should be better than TBW alone [18-20]. Curtis, in a cadaveric study on the strength of internal fixation for a fractured patella, compared Müller's TBW technique to the Pyrford technique using a cerclage wire and an anterior TBW looping through the quadriceps tendon [21, 22]. He found that all patellae fixed using Müller's technique failed at an applied force of $20 \mathrm{~kg}$ or less and none of the specimens fixed by the Pyrford technique failed at $25 \mathrm{~kg}$ [22]. Accordingly, we proposed a null hypothesis that concurrent application of an ACCW does not help fracture stabilization and enhance fracture healing in patients having been treated by TBW for their displaced transverse fractures of the body of the patella. Through a retrospective cohort study design, we tested the hypothesis based on the data (evidence) collected and then decided to either reject the null hypothesis or failed to reject it. The purpose of this study was (1) to determine the impact of an ACCW on the radiographic outcomes and (2) to explore the prognostic factors predicting treatment failure (e.g., fixation failure) in these patients.

\section{Methods}

Study population and primary/secondary endpoints

This retrospective cohort study included all patients with a diagnosis of displaced transverse fractures of the patella (AO/OTA type 34-C1.1 and 34-C2) who were treated by modified TBW [15] at our institution between January 2006 and June 2012. After obtaining approval from the Institutional Review Board, cases were identified by matching the International Classification of Diseases (ICD), 9th Revision codes specific for closed patella fracture (822.0) in the computerized trauma registry database of the hospital. At this stage, 185 patients with an acute patella fracture were identified and their medical records were reviewed and confirmed by two independent researchers (TYY and $\mathrm{KCH}$ ). Among them, we excluded patients aged $<18$ years, lost to follow up within 3 months after surgery and those with inadequate radiographs, bilateral patella fractures, open or multiple fractures, vertical fractures, complex fractures, and avulsion fractures of the upper or lower pole of the patella. The aggregate number of enrolled patients in this 6.5-year study was 72 .

We summarized the collected data at the time of study enrollment, which included demographics (age and gender), AO/OTA fracture types, surgical methods, aftercare program, radiographic results and surgical complications. To clarify the role of an ACCW on treatment outcomes of displaced transverse patella fracture, we classified the enrolled subjects into two groups for further analyses: fractures treated with modified TBW and ACCW (group 1, $n=27$ ) and those with modified TBW alone (group 2, $n=45$ ). The primary endpoint of this study was to determine the impact of an ACCW on the radiographic results of displaced transverse patella fracture having been treated by modified TBW. For our secondary endpoint, the effect of potential risk factors on fixation failure was analyzed by subgroup comparisons.

\section{Definitions \\ Level of the K-wires}

It was categorized as superficial or deep. Superficial level was defined as the level of the K-wires anterior to the center of the patella. In contrast, we defined the deep level as being in the center of deeper position of the patella.

\section{Distance between the K-wires}

It was categorized as narrow or wide. Narrow distance was defined as the distance between the K-wires less than one third of the patella width. In contrast, we defined the wide distance as being equal or more than one third of the patella width.

\section{Fixation failure}

It was defined as hardware breakage, nonunion, or redisplacement of fragments from their initial reduced position with gap separation $>2 \mathrm{~mm}$. 


\section{Statistical analysis}

Variables were compared using univariate analysis. A $X^{2}$ analysis or a Fisher's exact test was used where appropriate for analyzing categorical data. For numerical data, the Wilcoxan rank sum test was utilized for between-group comparisons. Significance was set at $P<0.05$ (two-sided). Not statistically significant was abbreviated as NS. All statistic analyses were conducted using the Statistical Package for the Social Sciences (SPSS, version 12.0. SPSS Inc., Chicago, IL, USA) and/or the MedCalc Statistical Software (version 9.5, Broekstraat, Mariakerke, Belgium).

\section{Results}

According to the inclusion/exclusion criteria, 72 (38.9\%) of 185 episodes were eligible for this study and divided into two groups, including the group of fractures treated with modified TBW and ACCW (group 1, $n=27$ ) and the group of those treated with modified TBW alone (group 2, $n=45$ ). Baseline data on these 72 patients treated with modified TBW for displaced transverse fractures of the patella are shown in Table 1. Age, age distribution, gender, fracture pattern, level of the K-wires, distance between the K-wires, and aftercare such as use of postoperative splint

Table 1 Characteristics of patients treated by tension band wiring technique with or without augmented cerclage wire fixation

\begin{tabular}{|c|c|c|c|}
\hline Variables & Group $1(n=27)$ & Group $2(n=45)$ & $P$ Value \\
\hline Age (years), mean (range) & $61.33(32-87)$ & $63.73(25-88)$ & 0.530 \\
\hline \multicolumn{3}{|l|}{ Age > 60 years, $\mathrm{n}(\%)$} & 0.341 \\
\hline Yes & $13(48.1)$ & $27(60.0)$ & \\
\hline No & $14(51.9)$ & $18(40.0)$ & \\
\hline \multicolumn{3}{|l|}{ Age > 70 years, $n(\%)$} & 0.807 \\
\hline Yes & $11(40.7)$ & $21(46.7)$ & \\
\hline No & $16(59.3)$ & $24(53.3)$ & \\
\hline \multicolumn{3}{|l|}{ Gender, n (\%) } & 0.802 \\
\hline Male & $11(40.7)$ & $16(35.6)$ & \\
\hline Female & $16(59.3)$ & $29(64.4)$ & \\
\hline \multicolumn{3}{|l|}{ Fracture pattern, n (\%) } & 0.535 \\
\hline AO/OTA type 34-C2 & $6(22.2)$ & $7(15.6)$ & \\
\hline AO/OTA type 34-C1.1 & $21(77.8)$ & $38(84.4)$ & \\
\hline \multicolumn{3}{|l|}{ Level of the K-wires, n (\%) } & 0.308 \\
\hline Superficial & $7(25.9)$ & $18(40.0)$ & \\
\hline Deep & $20(74.1)$ & $27(60.0)$ & \\
\hline \multicolumn{3}{|c|}{ Distance between the K-wires, $\mathrm{n}(\%)$} & 0.085 \\
\hline Narrow & $12(44.4)$ & $30(66.7)$ & \\
\hline Wide & $15(55.6)$ & $15(33.3)$ & \\
\hline \multicolumn{3}{|l|}{ Postoperative splinting, n (\%) } & 0.807 \\
\hline Yes & $16(59.3)$ & $24(53.3)$ & \\
\hline No & $11(40.7)$ & $21(46.7)$ & \\
\hline
\end{tabular}

${ }^{*} P$ value $<0.05$ is significant did not differ between the two groups of patients. Regarding the outcome analysis, there were no significant differences in the rates of fixation failure $(P=0.620)$, nonunion $(P=0.620)$, or revision surgery $(P=0.620)$ between the groups (Table 2$)$. In total there were 4 patients $(5.6 \%)$ having fixation failure, including 2 group- 1 patients (7.4\%) and 2 group-2 patients (4.4\%), respectively. All of them had nonunion but no wire migration and needed revision surgery to restore their extensor mechanism and enhance fracture union. After bone grafting and revision modified TBW surgery focusing on the adjustment of the level of the K-wires and the distance between them, all these fixation failure patients recovered their function of extensor mechanism and had their patella fractures united eventually (Table 3).

Several potential risk factors for fixation failure were analyzed and shown in Table 4. Although not statistically significant, we observed a trend of positive risk association between fixation failure and age distribution $>60$ years $(10.0 \%$ vs. $0.0 \%, P=0.124$; $\mathrm{OR}=8.0, P=0.168)$ and $>70$ years $(9.4 \%$ vs. $2.5 \%, P=$ 0.321 ; $\mathrm{OR}=4.0, P=0.237$ ) and the superficial level of the K-wires $(12.0 \%$ vs. $2.1 \%, P=0.117 ; \mathrm{OR}=6.3, P=0.121)$. Regarding those patients concurrently treated with an ACCW, the risk association between fixation failure and the superficial level of the K-wire increased further $(28.6 \%$ vs. $0.0 \%, P=0.060 ; \mathrm{OR}=18.6, P=0.071$ ).

\section{Discussion}

The decision of the current retrospective cohort study was failure to reject the null hypothesis [23], i.e., there was no sufficient evidence supporting that the concurrent application of an ACCW could provide a better treatment outcome (e.g., less fixation failure) than modified TBW

Table 2 Radiographic results and revision surgery of patients treated by tension band wiring technique with or without augmented cerclage wire fixation

\begin{tabular}{|c|c|c|c|}
\hline Variables & Group $1(n=27)$ & Group $2(n=45)$ & $P$ Value \\
\hline Fixation failure, n (\%) & & & 0.620 \\
\hline Yes & $2(7.4)$ & $2(4.4)$ & \\
\hline No & $25(92.6)$ & $43(95.6)$ & \\
\hline Nonunion, n (\%) & & & 0.620 \\
\hline Yes & $2(7.4)$ & $2(4.4)$ & \\
\hline No & $25(92.6)$ & $43(95.6)$ & \\
\hline Wire migration, n (\%) & & & 1.000 \\
\hline Yes & $0(0.0)$ & $0(0.0)$ & \\
\hline No & $27(100.0)$ & $45(100.0)$ & \\
\hline Revision surgery, n (\%) & & & 0.620 \\
\hline Yes & $2(7.4)$ & $2(4.4)$ & \\
\hline No & 25 (92.6) & $43(95.6)$ & \\
\hline
\end{tabular}

${ }^{*} P$ value $<0.05$ is significant 
Table 3 Demographic data of the cases with fixation failure

\begin{tabular}{llllll}
\hline Variables & \multicolumn{2}{l}{ Group 1 } & & Group 2 & \\
\cline { 2 - 3 } \cline { 5 - 6 } & Patient 1 & Patient 2 & & Patient 3 & Patient 4 \\
\hline Age, years & 66 & 78 & 73 & 88 \\
Gender & F & M & F & M \\
AO/OTA type 34-C2 fracture & N & N & N & N \\
Superficial level of the K-wires & Y & Y & N & Y \\
Narrow K-wire distance & Y & N & Y & N \\
Without postoperative & Y & N & Y & N \\
splinting & & & & \\
K-wire migration & N & N & N & N \\
Revision surgery & Y & Y & Y & Y \\
Final union & Y & Y & Y & Y \\
\hline
\end{tabular}

${ }^{*} P$ value $<0.05$ is significant

alone while treating patients with displaced transverse fractures of the patella. The fixation failure rate in group 1 and group 2 patients was 7.4 and 4.4\%, respectively $(P=\mathrm{NS})$. All these fixation failure patients recovered their function of extensor mechanism and had their patella fractures united by bone grafting and revision surgery with modified TBW alone, focusing on the adjustment of the level of the K-wires and the distance between them. Although not statistically significant, prognostic factor analyses showed that there was a trend towards a higher rate of fixation failure in elderly patients $(\mathrm{OR} \geq 4.0, P=\mathrm{NS})$ and those with their K-wires at the superficial level $(\mathrm{OR}=6.3, P=\mathrm{NS})$. Concurrently applying an ACCW did not decrease the risk association between fixation failure and the superficial level of the K-wires $(\mathrm{OR}=18.6$ and 1.5 , both $P=$ NS, in group 1 and group 2 patients, respectively). We, thus, highlight that concurrent application of an ACCW in patients having been treated with modified TBW technique might be not as efficacious as what we had learned from the literature [18-20]. Reviewing the literature showed that all of these clinical articles were categorized as case-series, a study design lacking comparison groups and thus vulnerable to selection bias and easily leading us to an incorrect conclusion $[24,25]$. To further clarify the role and effect of concurrent application of an ACCW on the treatment

Table 4 Prognostic factor analyses: subgroup comparisons of the effects of patient characteristics on fixation failure

\begin{tabular}{|c|c|c|c|c|c|}
\hline Variables & Fixation Failure & Union & $P$ Value & OR $(95 \% \mathrm{Cl})$ & $P$ Value \\
\hline All patients, n (\%) & $(n=4)$ & $(n=68)$ & & & \\
\hline Patient age $>60$ years & $4(100.0)$ & $36(52.9)$ & 0.124 & $8.0(0.4-154.6)$ & 0.168 \\
\hline Patient age $>70$ years & $3(75.0)$ & $29(42.6)$ & 0.321 & $4.0(0.4-40.8)$ & 0.237 \\
\hline Female patients & $2(50.0)$ & $43(63.2)$ & 0.628 & $0.6(0.1-4.4)$ & 0.599 \\
\hline AO/OTA type 34 C2 fracture & $0(0.0)$ & $13(19.1)$ & 1.000 & $0.5(0.0-9.0)$ & 0.607 \\
\hline Superficial level of the K-wires & $3(75.0)$ & $22(32.3)$ & 0.117 & $6.3(0.6-63.8)$ & 0.121 \\
\hline Narrow K-wire distance & $2(50.0)$ & $40(58.8)$ & 1.000 & $0.7(0.1-5.3)$ & 0.729 \\
\hline Without postoperative splinting & $2(50.0)$ & $30(44.1)$ & 1.000 & $1.3(0.2-9.5)$ & 0.818 \\
\hline Group 1 patients, n (\%) & $(n=2)$ & $(n=25)$ & & & \\
\hline Patient age $>60$ years & $2(100.0)$ & $11(44.0)$ & 0.222 & $6.3(0.3-144.7)$ & 0.249 \\
\hline Patient age $>70$ years & $1(50.0)$ & $10(40.0)$ & 1.000 & $1.5(0.1-26.9)$ & 0.783 \\
\hline Female patients & $1(50.0)$ & $15(60.0)$ & 1.000 & $0.1(0.0-3.1)$ & 0.212 \\
\hline AO/OTA type 34 C2 fracture & $0(0.0)$ & $6(24.0)$ & 1.000 & $0.6(0.0-14.2)$ & 0.752 \\
\hline Superficial level of the K-wires & $2(100.0)$ & $5(20.0)$ & 0.060 & $18.6(0.8-447.7)$ & 0.071 \\
\hline Narrow K-wire distance & $1(50.0)$ & $11(44.0)$ & 1.000 & $1.3(0.1-22.7)$ & 0.870 \\
\hline Without postoperative splinting & $1(50.0)$ & $10(40.0)$ & 1.000 & $1.5(0.1-26.9)$ & 0.783 \\
\hline Group 2 patients, n (\%) & $(n=2)$ & $(n=43)$ & & & \\
\hline Patient age $>60$ years & $2(100.0)$ & $25(58.1)$ & 0.509 & $3.6(0.2-80.1)$ & 0.415 \\
\hline Patient age $>70$ years & $2(100.0)$ & $19(44.2)$ & 0.212 & $6.3(0.3-138.6)$ & 0.244 \\
\hline Female patients & $1(50.0)$ & $28(65.1)$ & 1.000 & $0.5(0.0-9.2)$ & 0.667 \\
\hline AO/OTA type 34 C2 fracture & $0(0.0)$ & $7(16.3)$ & 1.000 & $1.0(0.0-22.4)$ & 0.987 \\
\hline Superficial level of the K-wires & $1(50.0)$ & $17(39.5)$ & 1.000 & $1.5(0.1-26.1)$ & 0.769 \\
\hline Narrow K-wire distance & $1(50.0)$ & $29(67.4)$ & 1.000 & $0.5(0.0-8.3)$ & 0.616 \\
\hline Without postoperative splinting & $1(50.0)$ & $20(66.7)$ & 1.000 & $1.2(0.1-19.6)$ & 0.923 \\
\hline
\end{tabular}

*P value $<0.05$ is significant 
outcomes, a double-blinded prospective randomized controlled trial is, therefore, necessary in the future.

To the best of our knowledge, this is the first report to determine the impact of concurrently applying an ACCW on the treatment outcomes of displaced transverse patella fractures having been fixed by modified TBW. The present study also provides a higher level of evidence than that of previously published articles by others [18-20]. Moreover, our results challenge the concept proposed and propelled by Curtis in 1990 [22]. After comparing Pyrford technique (TBW + ACCW) to Müller's technique (TBW alone) in a cadaveric study, Curtis concluded that the former gave greater strength of fixation and should be recommended. However, nearly all patellae fixed using Müller's TBW technique failed when the distal ends of the K-wire bent, which is also true in clinical scenario [26-29]. Because of the difficulty capturing the tension band by the migrated or bent distal ends of the K-wires, loss of fixation or reduction can occur in as many as $20 \%$ of patella fractures treated with Müller's TBW technique [26-29]. Many revised techniques and technologies were, therefore, developed to solve this problem [15, 18-20, 27-30]. Wu's modified TBW technique is one of the simplest and most effective methods [15]. Using this technique in this study, the total fixation failure rate was $5.6 \%$ only. It's interesting that concurrently applying an ACCW could not provide a superior result than Wu's modified TBW alone. Considering the potential hazards on patella vascularity and the non-superiority results, we, therefore, recommend that concurrent application of an ACCW may not be required. Adherence to correct surgical technique of modified TBW technique might be more important and help in improving outcomes.

Our results suggested that the level of the K-wires might be one of the most critical surgical pearls in the treatment of patients with displaced transverse fractures of the body of the patella. The TBW design for treating these lesions needs to handle compression and tension without buckling and snapping. When the compression force overcomes bone's ability to handle compression, buckling occur [31]. When the tension force overcomes wires' ability to handle tension, snapping occurs [26-29, 32-34]. The TBW technique should transfer and dissipate these forces. Putting the parallel K-wires at the superficial level (i.e., anterior to the center of the patella) and with a narrow distance between them (i.e., less then one third of the patellar width) might be inadequate to transfer and dissipate the compression force, thus resulting in buckling and cut-through phenomena [32]. Applying the TBW without securing control of the both ends of the K-wires might be inadequate to transfer and dissipate the tension force, thus resulting in snapping and wire migration, bending, or breakage [33, 34]. Our findings indicated that Wu's modified TBW technique could effectively control the both ends of the K-wires, thus eliminate the possibility of wire migration/bending/breakage and then improve the fixation stability. Concurrently applying an ACCW could not further help the current fixation design handle compression and tension forces. Instead, putting the K-wires at the deep level and with wide distance between them might be more helpful for handling these deforming forces.

Osteoporotic bone is at risk for fractures and fixation failure after surgical stabilization [35]. To determine anatomic sites and circumstances under which a fracture may be a consequence of osteoporosis, Warriner et al. developed the osteoporosis attribution scoring (OAS) system and provided an evidence-based continuum of the likelihood of a fracture being associated with osteoporosis. Using the OAS system they categorized patella fracture as a high likelihood of being because of osteoporosis in older Caucasian women but an indeterminate likelihood in younger African-American man [36]. Our results revealed that there was a paradoxical relationship between fixation failure in patella fractures and some osteoporosis risk factors (e.g., aging, gender and bone quality) $[37,38]$. We found a trend towards an increased risk of fixation failure in geriatric patients; however, the risk association did not increase with increasing age. Female gender, fracture comminution (AO/OTA type 34-C2) and without use of postoperative splint were not associated with an increased risk of fixation failure. Whatever role osteoporosis might play in the treatment of patella fractures, we believe that refinement of TBW technique and/or technology to successfully handle the deforming forces should be the most critical surgical pearls. The refined technique and/or technology would effectively improve fixation stability, permit early joint motion, enhance fracture healing, promise an excellent treatment outcome, and thus decrease the cost burden on the family and society for that condition.

Our study has several limitations. First, it is retrospective. Second, the number of patients with treatment failure included during the study period was relatively small and the study may have lacked power to detect the statistical differences in all prognostic factors among subsets of patients. The uncommon occurrence of fixation failure in the current study is to be expected because of the excellent results provided with Wu's modified TBW technique [15]. After a consecutive follow-up study lasting for more than 6 years, our results highlighted the importance of adherence to correct modified TBW technique instead of adding an ACCW in treating these patients. Third, there is lack of functional outcome measures in this study. For treatment of displaced transverse fractures of the patella, TBW technique has been a well-documented surgical method. The role of concurrently applying an ACCW to fractured patella having been fixed with TBW is intended to help fracture stabilization. The purpose of this study was therefore to 
determine the impact of concurrent application of an ACCW on the treatment outcomes (i.e., fixation failure) until bone healing, leaving functional outcome consideration aside. Lastly, only AO/OTA type 34-C1.1 or 34- $\mathrm{C} 2$ fractures were included in this investigation. We excluded all complex fractures and avulsion fractures of the proximal or distal pole of the patella in the current work in order to reduce systemic errors and bias to the smallest possible degree.

\section{Conclusion}

We concluded that there was no sufficient evidence supporting that the concurrent application of an ACCW could provide a better treatment outcome (e.g., less fixation failure) than Wu's modified TBW alone while treating patients with a displaced transverse fracture of the patella. Wu's modified TBW technique could effectively control the both ends of the K-wires, thus eliminate the possibility of wire migration/bending/breakage and then improve the fixation stability. Putting the K-wires at the deep level and with wide distance between them might effectively transfer and dissipate the compression force, thus decrease the chance of cut-through phenomena and loss of fixation/reduction. Considering the potential hazards on patella vascularity and the non-superiority results, we, therefore, recommend that concurrent application of an ACCW may not be required.

\section{Abbreviations}

ACCW: Augmented circumferential cerclage wire; AO/OTA: Arbeitsgemeinschaft für Osteosynthesefragen/Orthopaedic Trauma Association; ICD: International Classification of Diseases; K-wire: Kirschner-wire; NS: not significant; OAS: Osteoporosis Attribution Scoring; OR: odds ratio; SPSS: Statistical Product and Service Solutions; STROBE: Strengthening the Reporting of Observational studies in Epidemiology; TBW: tension band wire

\section{Acknowledgements}

We would like to thank Wen-Chi Liang for her assistance with data collection and statistical analysis.

\section{Ethic approval and consent to participate}

The authors analyzed the datas after approval by the ethic committee (Institutional Review Board) of the Chang Gung Memorial Hospital in Taiwan (Reference number: 102-0252B). We did not obtain informed consent from the patient due to a statement of this committee, that analyzing patient data retrospectively requires no informed consent. However, patient records and information were anonymized and de-identified prior to analysis.

\section{Availability of data and materials}

All data and information about materials are in the paper and/or available from the first author

\section{Authors' contributions \\ TYY and $\mathrm{KCH}$ were involved in the study conception and design, data acquisition, analysis, interpretation, and drafting the manuscript. TWH and PYC were involved in data collection, critical review and analysis of results, and editing the manuscript. All authors read and approved the final manuscript.}

\section{Competing interests}

The authors declare that they have no competing interests.

\section{Publisher's Note}

Springer Nature remains neutral with regard to jurisdictional claims in published maps and institutional affiliations.

\section{Author details}

${ }^{1}$ Chang Gung University College of Medicine, Taoyuan, Taiwan. ${ }^{2}$ Department of Orthopaedic Surgery, Chang Gung Memorial Hospital, Chiayi, Taiwan.

${ }^{3}$ Putz City, Taiwan.

Received: 9 May 2017 Accepted: 15 May 2018

Published online: 24 May 2018

\section{References}

1. Nummi J. Fracture of the patella. A clinical study of 707 patellar fractures. Ann Chir Gynaecol Fenn Suppl. 1971;179:1-85.

2. Melvin JS, Metha S. Patellar fractures in adults. J Am Acad Orthop Surg. 2011:19(4):198-207.

3. Mao N, Ni H, Ding W, Zhu X, Bai Y, Wang C, et al. Surgical treatment of transverse patella fractures by the cable pin system with a minimally invasive technique. J Trauma Acute Care Surg. 2012;72(4):1056-61.

4. Yang NP, Chan CL, Yu IL, Lee CY, Chou P. Estimated prevalence of orthopaedic fractures in Taiwan: a cross-sectional study based on nationwide insurance data. Injury. 2010;41(12):1266-72.

5. Yamaguchi GT, Zajac FE. A planar model of the knee joint to characterize the knee extensor mechanism. J Biomech. 1989;22(1):1-10.

6. Greisamer RP, Weinstein H. Applied biomechanics of the patella. Clin Orthop Relat Res. 2001;389:9-14.

7. Hung LK, Chan KM, Chow YN, Leung PC. Fractured patella: operative treatment using the tension band principle. Injury. 1985;16(5):343-7.

8. Weber MJ, Janecki CJ, McLeod P, Nelson CL, Thompson JA. Efficacy of various forms of fixation of transverse fractures of the patella. J Bone Joint Surg Am. 1980;62(2):215-20.

9. Chalidis BE, Sachinis NC, Samoladas EP, Dimitriou CG, Pournaras JD. Is tension band wiring technique the "gold standard" for the treatment of olecranon fractures? A long term functional outcome study. J Orthop Surg Res. 2008:3:9.

10. Maranho DA, Pagnano RG, Volpon JB. Tension band wiring for proximal femoral varus osteotomy fixation in children. Medicine (Baltimore). 2014:93(7):e61.

11. Stoffel K, Klaue K, Perren SM. Functional load of plates in fracture fixation in vivo and its correlate in bone healing. Injury. 2000;31(Suppl 2):S-B37-50.

12. Patel VR, Parks $B G$, Wang $Y$, Ebert FR, Jinnah RH. Fixation of patella fractures with braided polyester suture: a biomechanical study. Injury. 2000;31(1):1-6.

13. Baran O, Manisali M, Cecen B. Anatomical and biomechanical evaluation of the tension band technique in patellar fractures. Int Orthop. 2009;33(4): $1113-7$.

14. Lotke PA, Ecker ML. Transverse fractures of the patella. Clin Orthop Relat Res. 1981;158:180-4.

15. Wu CC, Tai CL, Chen WJ. Patellar tension band wiring: a revised technique. Arch Orthop Trauma Surg. 2001;121(1-2):12-6.

16. Hoshino CM, Tran W, Tiberi JV, Black MH, Li BH, Gold SM, et al. Complications following tension-band fixation of patellar fractures with cannulated screws compared with Kirschner wire. J Bone Joint Surg Am. 2013;95(7):653-9.

17. Taylor BC, Mehta S, Castaneda J, French BG, Blanchard C. Plating of patella fractures: techniques and outcomes. J Orthop Trauma. 2014;28(9):e231-5.

18. Agarwala S, Agrawal P, Sobti A. A novel technique of patella fracture fixation facilitating early mobilization and reducing re-operation rates. J Clin Orthop Trauma. 2015:6(3):207-11.

19. Ong TK, Chee EK, Wong CL, Thevarajan K. Fixation of comminuted patellar fracture with combined cerclage and tension band wiring technique. Malaysian Orthop J. 2008;2(2):40-2.

20. Us AK, Kinik H. Self locking tension band technique in transverse patellar fracture. Int Orthop. 1996;20(6):357-8.

21. Müller ME, Allgöwer M, Schneider R, Willenegger H. Manual of internal fixation: techniques recommended by the AO group, Berlin, etc. SpringerVerlag, 2nd edition, 1979.

22. Curtis MJ. Internal fixation for fractures of the patella. A comparison of two methods. J Bone Joint Surgery Br. 1990;72(2):280-2.

23. Lew MJ. Principles: when there should be no difference: how to fail to reject the null hypothesis. Trends in Pharmacol Sci. 2006;27(5):274-8. 
24. Abu-Zidan FM, Abbas AK, Hefny AF. Clinical "case series": a concept analysis. Afr Health Sci. 2012;12(4):557-62.

25. Schaafsma F, Verbeek J, Hulshof C, van Dijk F. Caution required when replying on a colleague's advice; a comparison between professional advice and evidence from the literature. BMC Health Serv Res. 2005;5:59.

26. Smith ST, Cramer KE, Karges DE, Watson JT, Moed BR. Early complications in the operative treatment of patella fractures. J Orthop Trauma. 1997;11(3):183-7.

27. Tian Y, Zhou F, Ji H, Zhang Z, Guo Y. Cannulated screw and cable are superior to modified tension band in the treatment of transverse patella fractures. Clin Orthop Relat Res. 2011;469(12):3429-35.

28. Wang CX, Tan L, Qi BC, Hou XF, Huang YL, Zhang HP, et al. A retrospective comparison of the modified tension band technique and the parallel titanium annulated lag screw technique in transverse patella fracture. Chin J Traumatol. 2014;17(4):208-13.

29. Lin T, Liu J, Xiao B, Fu D, Yang S. Comparison of the outcomes of cannulated screws vs. modified tension band wiring fixation techniques in the management of mildly displaced patellar fractures. BMC Musculoskelet Disord. 2015;16(1):282

30. Lefaivre KA, O'Brien PJ, Broekhuyse HM, Guy P, Blachut PA. Modified tension band technique for patella fractures. Orthop Traumatol Surg Res. 2010;96(5): 579-82.

31. Nagaraja S, Couse TL, Guldberg RE. Trabecular bone microdamage and microstructural stresses under uniaxial compression. J Biomech. 2005;38(4):707-16.

32. Huang TW, Hsieh PH, Huang KC, Huang KC. Suspension suture augmentation for repair of coracoclavicular ligament disruption. Clin Orthop Relat Res. 2009;467(8):2142-8.

33. Mullett JH, Shannon F, Noel J, Lawlor G, Lee TC, O'Rourke SK. K-wire position in tension band wiring of the olecranon: a comparison of two techniques. Injury. 2000;31(6):427-31.

34. Saeed ZM, Trickett RW, Yewlett AD, Matthews TJ. Factors influencing K-wire migration in tension-band wiring of olecranon fractures. J Shoulder Elb Surg. 2014;23(8):1181-6.

35. Cornell CN. Internal fracture fixation in patients with osteoporosis. J Am Acad Orthop Surg. 2003;11(2):109-19.

36. Warriner AH, Patkar NM, Curtis JR, Delzell E, Gary L, Kilgore M, et al. Which fractures are most attributable to osteoporosis? J Clin Epidemiol. 2011;64(1): 46-53.

37. Miller MA, Liu W, Zurakowski D, Smith RM, Harris MB, Vrahas MS. Factors predicting failure of patella fixation. J Trauma Acute Care Surg. 2012;72(4): $1051-5$.

38. Böstmann O, Kiviluoto O, Nirhamo J. Comminuted displaced fractures of the patella. Injury. 1981;13(3):196-202.

\section{Ready to submit your research? Choose BMC and benefit from:}

- fast, convenient online submission

- thorough peer review by experienced researchers in your field

- rapid publication on acceptance

- support for research data, including large and complex data types

- gold Open Access which fosters wider collaboration and increased citations

- maximum visibility for your research: over $100 \mathrm{M}$ website views per year 